\title{
ESTIMATES FOR EIGENVALUES OF THE SCHRÖDINGER OPERATOR WITH A COMPLEX POTENTIAL
}

\author{
OLEG SAFRONOV
}

\begin{abstract}
We study the distribution of eigenvalues of the Schrödinger operator with a complex valued potential $V$. We prove that if $|V|$ decays faster than the Coulomb potential, then all eigenvalues are in a disc of a finite radius.
\end{abstract}

\section{INTRODUCTION}

We consider the Schrödinger operator $H=-\Delta+V$ with a complex potential $V$ and then we study the distribution of eigenvalues of $H$ in the complex plane.

Our work in this direction was motivated by the question of E.B. Davies about an integral estimate for eigenvalues of $H$ (see [1] and [2]). If $d=1$ then all eigenvalues of $H$ which do not belong to $\mathbb{R}_{+}=[0, \infty)$ satisfy

$$
|\lambda| \leq \frac{1}{4}\left(\int_{\mathbb{R}}|V(x)| d x\right)^{2} .
$$

The question is whether something similar holds in dimension $d \geq 2$. We prove the following result related directly to this matter.

Theorem 1.1. Let $V: \mathbb{R}^{d} \mapsto \mathbb{C}$ satisfy the condition

$$
|V(x)| \leq \frac{L}{\left(1+|x|^{2}\right)^{p / 2}}, \quad 1<p<3,
$$

with a constant $L>0$. Let $\varkappa=(p-1) / 2$ and let $\epsilon>0$ be an arbitrarily small number that belongs to the intersection of the intervals $(0,(1-\varkappa) / 2) \cap$ $(0,1 / 2)$. Then any eigenvalue $\lambda \notin \mathbb{R}_{+}$of $H$ with $\Re \lambda>0$ satisfies one of the conditions:

$$
\text { 1) either }|\lambda| \leq 1
$$

or

$$
\text { 2) } 1 \leq C L\left(|\Re \lambda|^{(\varkappa+2 \varepsilon-1) / 2}+|\lambda|^{\varepsilon-1 / 2}+\frac{1+|\lambda|^{\varepsilon}}{(|\lambda|-1)}\right)
$$

where the constant $C$ depends on the dimension $d$ and on the parameters $p$ and $\varepsilon$. In particular, it means that all non-real eigenvalues are in a disc of a finite radius.

2000 Mathematics Subject Classification. $47 \mathrm{~F} 05$.

Key words and phrases. Eigenvalue estimates, complex potentials, Schrödinger operators. 
The study of eigenvalue estimates for operators with a complex potential already has a bibliography. Besides [1] and [2], we would like to mention the papers [3] and [4]. The main result of [3] tells us, that for any $t>0$, the eigenvalues $z_{j}$ of $H$ lying outside the sector $\{z:|\Im z|<t \Re z\}$ satisfy the estimate

$$
\sum\left|z_{j}\right|^{\gamma} \leq C \int|V(x)|^{\gamma+d / 2} d x, \quad \gamma \geq 1
$$

where the constant $C$ depends on $t, \gamma$ and $d$ (see also [5] for the case when $V$ is real).

The paper [4] deals with natural question that appears in relation to the main result of [3]: what estimates are valid for the eigenvalues situated inside the conical sector $\{z:|\Im z|<t \Re z\}$, where the eigenvalues might be close to the positive half-line? Theorems of the article [4] provide some information about the rate of accumulation of eigenvalues to the set $\mathbb{R}_{+}=[0, \infty)$. Namely, [4] gives sufficient conditions on $V$ that guarantee convergence of the sum

$$
\sum_{a<\Re z_{j}<b}\left|\Im z_{j}\right|^{\gamma}<\infty
$$

for $0 \leq a<b<\infty$. Moreover, the following result is also proven in [4]:

Theorem 1.2. Let $V$ be a function from $L^{p}\left(\mathbb{R}^{d}\right)$, where $p \geq d / 2$, if $d \geq 3 ;$; $p>1$, if $d=2$, and $p \geq 1$, if $d=1$. Then every eigenvalue $\lambda$ of the operator $H=-\Delta+V$ with the property $\Re \lambda>0$ satisfies the estimate

$$
|\Im \lambda|^{p-1} \leq|\lambda|^{d / 2-1} C \int_{\mathbb{R}^{d}}|V|^{p} d x
$$

The constant $C$ in this inequality depends only on $d$ and $p$. Moreover, $C=$ $1 / 2$ for $p=d=1$.

\section{Proof of Theorem 1.1}

Consider first the case $L=1$. For the sake of convenience we introduce the notations $W=|V|^{1 / 2}$ and $l=p / 2$. According to the Birman-Schwinger principle, a number $\lambda \notin \mathbb{R}_{+}$is an eigenvalue of the operator $H=-\Delta+V(x)$ if and only if the number -1 is an eigenvalue of the operator

$$
X_{0}=W(-\Delta-\lambda)^{-1} W \frac{V}{|V|} .
$$

Therefore if $\lambda$ is a point of the spectrum of the operator $H$, then $\left\|X_{0}\right\| \geq 1$. On the other side, since multiplication by the function $\frac{V}{|V|}$ represents a unitary operator, the condition $\left\|X_{0}\right\| \geq 1$ implies that the norm of the operator

$$
X=W(-\Delta-\lambda)^{-1} W
$$

is also not less than 1 . 
In order to estimate the norm of the operator $X$ from above, we consider its kernel

$$
(2 \pi)^{-d} W(x) \int \frac{e^{i \xi(x-y)}}{\xi^{2}-\lambda} d \xi W(y)
$$

It follows from this formula that $X$ can be represented in the form

$$
X=\int_{0}^{\infty} \frac{\Gamma_{\rho}^{*} \Gamma_{\rho}}{\rho^{2}-\lambda} d \rho
$$

where $\Gamma_{\rho}$ is the operator mapping $L^{2}\left(\mathbb{R}^{d}\right)$ into $L^{2}\left(\mathbb{S}_{\rho}\right)$, and $\mathbb{S}_{\rho}$ is the sphere of radius $\rho$ with the center at the point 0 :

$$
\Gamma_{\rho} u(\theta)=(2 \pi)^{-d / 2} \int_{\mathbb{R}^{d}} e^{-i \rho(\theta x)} W(x) u(x) d x
$$

The main properties of this operator follow from Sobolev's embedding theorems. Suppose that $W(x) \leq\left(1+|x|^{2}\right)^{-l / 2}$ and $u \in L^{2}\left(\mathbb{R}^{d}\right)$. Then the Fourier transformation of the function $W(x) u(x)$ belongs to the class $H^{l}\left(\mathbb{R}^{d}\right)$, moreover the norm $\|\hat{W} u\|_{H^{l}}$ is estimated by the norm $\|u\|_{L^{2}}$. According to Sobolev's theorems, the embedding of the class $H^{l}\left(\mathbb{R}^{d}\right)$ into the class $L^{2}\left(\mathbb{S}_{\rho}\right)$ is continuous under the condition $l>1 / 2$. Moreover, the norm of the embedding operator depends in a weak manner on the parameter $\rho \geq 1$. Indeed, suppose that the inequality

$$
\int_{\mathbb{S}_{1}}|\phi(\theta)|^{2} d \theta \leq C \int_{\mathbb{R}^{d}}\left(\left|\nabla^{l} \phi\right|^{2}+|\phi|^{2}\right) d x
$$

holds for any function $\phi \in H^{l}\left(\mathbb{R}^{d}\right)$. Then setting $\phi(x)=u(\rho x)$ we obtain that

$$
\int_{\mathbb{S}_{1}}|u(\rho \theta)|^{2} d \theta \leq C \int_{\mathbb{R}^{d}}\left(\rho^{2 l}\left|\nabla^{l} u(\rho x)\right|^{2}+|u(\rho x)|^{2}\right) d x .
$$

Multiplying both sides of this inequality by $\rho^{d-1}$, we obtain that

$$
\int_{\mathbb{S}_{\rho}}|u(x)|^{2} d S \leq C \int_{\mathbb{R}^{d}}\left(\rho^{2 l-1}\left|\nabla^{l} u(x)\right|^{2}+\rho^{-1}|u(x)|^{2}\right) d x .
$$

If $l$ is close to $1 / 2$ then $\rho^{2 l-1}$ practically behaves as a constant. Anyway, without loss of generality we can assume that for $\rho>1$

$$
\int_{\mathbb{S}_{\rho}}|u(x)|^{2} d S \leq C_{\varepsilon} \rho^{2 \varepsilon}\|u\|_{H^{l}}^{2}
$$

where $\varepsilon>0$ is an arbitrary small number. It implies that

$$
\left\|\Gamma_{\rho}\right\| \leq C_{\varepsilon} \rho^{\varepsilon}, \quad \rho \geq 1
$$

Moreover, $\Gamma_{\rho}$ depends continuously on the parameter $\rho$ in the following sense. Let us introduce the operator $U_{\rho}$ that transforms functions on the sphere $\mathbb{S}_{\rho}$ into functions on the sphere $\mathbb{S}=\mathbb{S}_{1}$. according to the rule

$$
U_{\rho} u(\theta)=u(\rho \theta) \rho^{(d-1) / 2} .
$$


This operator is unitary and therefore its norm equals 1 . Define now the operator $Y_{\rho}=U_{\rho} \Gamma_{\rho}$. Our statement is that

$$
\left\|Y_{\rho^{\prime}}-Y_{\rho}\right\| \leq C\left|\rho^{\prime}-\rho\right|^{\alpha} \rho^{\delta}\left(\rho^{\varepsilon}+\left(\rho^{\prime}\right)^{\varepsilon}\right)
$$

where $\alpha<l-1 / 2, \delta=l-\alpha-1 / 2$ and $\rho^{\prime}>\rho \geq 1$. Our arguments are similar to those we used in the proof of the inequality (2.1). If we assume that the inequality

$$
\int_{\mathbb{S}_{1}}|\phi((1+h) \theta)-\phi(\theta)|^{2} d \theta \leq C h^{2 \alpha} \int_{\mathbb{R}^{d}}\left(\left|\nabla^{l} \phi\right|^{2}+|\phi|^{2}\right) d x
$$

holds for any function $\phi \in H^{l}\left(\mathbb{R}^{d}\right)$. Then the substitution $\phi(x)=u(\rho x)$ will lead to the inequality

$$
\int_{\mathbb{S}_{1}}|u((1+h) \rho \theta)-u(\rho \theta)|^{2} d \theta \leq C h^{2 \alpha} \int_{\mathbb{R}^{d}}\left(\rho^{2 l}\left|\nabla^{l} u(\rho x)\right|^{2}+|u(\rho x)|^{2}\right) d x .
$$

Multiplying both sides of this inequality by $\rho^{d-1}$ and denoting $\rho^{\prime}=(1+h) \rho$, we obtain that

$$
\int_{\mathbb{S}_{\rho}}\left|u\left(\rho^{-1} \rho^{\prime} x\right)-u(x)\right|^{2} d S \leq C\left|\rho^{\prime}-\rho\right|^{2 \alpha} \int_{\mathbb{R}^{d}}\left(\rho^{2 \delta}\left|\nabla^{l} u(x)\right|^{2}+\rho^{-2 l}|u(x)|^{2}\right) d x .
$$

provided that $\rho^{\prime}>\rho \geq 1$. This leads to

$$
\left\|\left(\frac{\rho}{\rho^{\prime}}\right)^{(d-1) / 2} Y_{\rho^{\prime}}-Y_{\rho}\right\| \leq C\left|\rho^{\prime}-\rho\right|^{\alpha} \rho^{\delta} .
$$

We apply now the triangle inequality to estimate the norm of the difference $Y_{\rho^{\prime}}-Y_{\rho}$ for $\rho^{\prime}>\rho \geq 1$

$$
\left\|Y_{\rho^{\prime}}-Y_{\rho}\right\| \leq\left|\left(\frac{\rho}{\rho^{\prime}}\right)^{(d-1) / 2}-1\right||| Y_{\rho^{\prime}}||+C\left|\rho^{\prime}-\rho\right|^{\alpha} \rho^{\delta} \leq C_{0}\left(\rho^{\varepsilon}+\left(\rho^{\prime}\right)^{\varepsilon}\right)\left|\rho^{\prime}-\rho\right|^{\alpha} \rho^{\delta} .
$$

To be more convincing, we mention that

$$
\left|\left(\frac{\rho}{\rho^{\prime}}\right)^{(d-1) / 2}-1\right| \leq \min \left\{2^{-1}(d-1)\left|\rho^{\prime}-\rho\right|, 2\right\}
$$

Introduce now the notation $G_{\rho}=\Gamma_{\rho}^{*} \Gamma_{\rho}$. Obviously, $G_{\rho}$ aslo has representation $G_{\rho}=Y_{\rho}^{*} Y_{\rho}$. Consequently,

$$
\left\|G_{\rho^{\prime}}-G_{\rho}\right\| \leq\left\|Y_{\rho^{\prime}}^{*}-Y_{\rho}^{*}\right\| \cdot\left\|Y_{\rho^{\prime}}\right\|+\left\|Y_{\rho}^{*}\right\| \cdot\left\|Y_{\rho^{\prime}}-Y_{\rho}\right\| \leq C\left(\rho^{\varepsilon}+\left(\rho^{\prime}\right)^{\varepsilon}\right)^{2}\left|\rho^{\prime}-\rho\right|^{\alpha} \rho^{\delta} .
$$

Let us summarize the results. The operator $X$ can be written in the form

$$
X=\int_{0}^{\infty} \frac{G_{\rho} d \rho}{\rho^{2}-\lambda},
$$

where

$$
\left\|G_{\rho}\right\| \leq C \rho^{2 \varepsilon}, \quad \rho \geq 1
$$

and

$$
\left\|G_{\rho^{\prime}}-G_{\rho}\right\| \leq C\left(\rho^{\varepsilon}+\left(\rho^{\prime}\right)^{\varepsilon}\right)^{2}\left|\rho^{\prime}-\rho\right|^{\alpha} \rho^{\delta}, \quad \rho^{\prime}>\rho \geq 1
$$


Now, since the integral representation for the operator $X$ can be also rewritten in the form

$$
X=\int_{1}^{\infty} \frac{\left(G_{\rho}-G_{\tau}\right) d \rho}{\rho^{2}-\lambda}+\int_{1}^{\infty} \frac{G_{\tau} d \rho}{\rho^{2}-\lambda}+W(-\Delta-\lambda)^{-1} E[0,1] W
$$

where $\tau=|\Re \lambda|^{1 / 2}$ and $E[0,1]$ is the spectral projection of the operator $-\Delta$ corresponding to the interval $[0,1]$, we obtain that

$$
\|X\| \leq \int_{1}^{\infty} \frac{\| G_{\rho}-G_{\tau}|| d \rho}{\left|\rho^{2}-\lambda\right|}+\frac{\pi\left\|G_{\tau}\right\|}{2|\lambda|^{1 / 2}}+\frac{\|V\|_{L^{\infty}}+\left\|G_{\tau}\right\|}{(|\lambda|-1)}
$$

for $|\lambda|>1$. Consequently,

$$
\|X\| \leq C \int_{0}^{\infty} \frac{|\rho-\tau|^{\alpha}\left(\rho^{\delta}+|\Re \lambda|^{\delta / 2}\right)\left(\rho^{\varepsilon}+|\Re \lambda|^{\varepsilon / 2}\right)^{2}}{\left|\rho^{2}-\Re \lambda\right|} d \rho+C \frac{\tau^{2 \varepsilon}}{|\lambda|^{1 / 2}}+\frac{\|V\|_{L^{\infty}}+C \tau^{2 \varepsilon}}{(|\lambda|-1)}
$$

which leads to

$$
1 \leq\|X\| \leq C\left(|\Re \lambda|^{(\alpha+\delta+2 \varepsilon-1) / 2}+|\lambda|^{\varepsilon-1 / 2}+\frac{1+|\lambda|^{\varepsilon}}{(|\lambda|-1)}\right) .
$$

We proved the statement of the theorem for the case $L=1$. If $L \neq 1$ then this inequality takes the form

$$
1 \leq C L\left(|\Re \lambda|^{(\alpha+\delta+2 \varepsilon-1) / 2}+|\lambda|^{\varepsilon-1 / 2}+\frac{1+|\lambda|^{\varepsilon}}{(|\lambda|-1)}\right) .
$$

The proof is completed.

\section{REFERENCES}

[1] Abramov, A.A., Aslanyan, A., Davies, E.B.: Bounds on complex eigenvalues and resonances. J. Phys. A 34, 57-72 (2001)

[2] Davies, E.B., Nath, J.: Schrdinger operators with slowly decaying potentials. J. Comput. Appl. Math. 148 (1), 1-28 (2002)

[3] Frank, Rupert L.; Laptev, Ari; Lieb, Elliott H.; Seiringer, Robert Lieb-Thirring inequalities for Schrödinger operators with complex-valued potentials. Lett. Math. Phys. 77 (2006), no. 3, 309-316.

[4] Laptev, A. and Safronov, O: Eigenvalue estimates for Schrödinger operators with complex potentials submitted.

[5] Lieb, E. H. and Thirring, W.:Inequalities for the moments of the eigenvalues of the Schrödinger Hamiltonian and their relation to Sobolev inequalities, in Studies in Mathematical Physics (Essays in Honor of Valentine Bargmann), 269-303. Princeton Univ. Press, Princeton, NJ, 1976.

E-mail address: osafrono@uncc.edu 